\title{
Profound systemic alteration of the immune phenotype and an immunoglobulin switch in Erdheim-Chester disease in 78 patients from a single center
}

\author{
Fleur Cohen Aubart, ${ }^{1 *}$ Lucie Poupel, ${ }^{2 *}$ Flora Saint-Charles, ${ }^{2}$ Frédéric Charlotte, ${ }^{3}$ Youssef \\ Arsafi, ${ }^{2}$ Eric Frisdal, ${ }^{2}$ Damien Roos-Weil, ${ }^{3}$ Jean-François Emile, ${ }^{4}$ Zahir Amoura, ${ }^{1}$ Maryse \\ Guerin, ${ }^{2}$ Philippe Lesnik, ${ }^{2}$ Julien Haroche ${ }^{1}$ and Wilfried Le Goff ${ }^{2}$
}

\begin{abstract}
'Sorbonne Université, Assistance Publique-Hôpitaux de Paris, Service de Médecine Interne 2, Centre National de Référence Maladies Systémiques Rares et Histiocytoses, Hôpital Pitié-Salpêtrière, Paris; ${ }^{2}$ Sorbonne Université, INSERM, Institute of Cardiometabolism and Nutrition (ICAN), UMR_S1166, Paris; ${ }^{3}$ Sorbonne Université, Assistance Publique-Hôpitaux de Paris, Service d'anatomopathologie, Hôpital Pitié-Salpêtrière, Paris and ${ }^{4} E A 4340$, Université Versailles-Saint Quentin, Assistance Publique-Hôpitaux de Paris, Hôpital Ambroise Paré, Département de Pathologie, Boulogne, France.
\end{abstract}

*FCA and LP contributed equally as co-first authors.

\author{
Correspondence: \\ Julien Haroche \\ Julien.haroche@psl.aphp.fr \\ Wilfried Le Goff \\ wilfried.le_goff@sorbonne-universite.fr \\ Received: $\quad$ April 30, 2021. \\ Accepted: $\quad$ October 6,2021 \\ Prepublished: October 14, 2021. \\ https://doi.org/10.3324/haematol.2021.279118 \\ (02022 Ferrata Storti Foundation \\ Haematologica material is published under a \\ CC BY-NC license (c) (1) \&
}

\begin{abstract}
Erdheim-Chester disease (ECD) is a rare, systemic, non-Langerhans cell histiocytosis neoplasm, which is characterized by the infiltration of $\mathrm{CD} 63^{+} \mathrm{CD1} \mathrm{a}^{-}$histiocytes in multiple tissues. The BRAF ${ }^{\mathrm{V} 600 \mathrm{E}}$ mutation is frequently present in individuals with ECD and has been detected in hematopoietic stem cells and immune cells from the myeloid and systemic compartments. Immune cells and pro-inflammatory cytokines are present in lesions, suggesting that ECD involves immune cell recruitment. Although a systemic cytokine T-helper-1-oriented signature has been reported in ECD, the immune cell network orchestrating the immune response in ECD has yet to be described. To address this issue, the phenotypes of circulating leukocytes were investigated in a large, single-center cohort of 78 patients with ECD and compared with those of a group of 21 control individuals. Major perturbations in the abundance of systemic immune cells were detected in patients with ECD, with decreases in circulating plasmacytoid, myeloid 1, and myeloid 2 dendritic cells, mostly in $B R A F^{\mathrm{V} 600 \mathrm{E}}$ carriers, in comparison with individuals in the control group. Similarly, marked decreases in blood Thelper, cytotoxic, and B-lymphocyte numbers were observed in patients with ECD, relative to the control group. Measurement of circulating immunoglobulin concentrations revealed an immunoglobulin $G$ switch, from IgG1 to IgG4 subclasses, which are more frequently associated with the BRAF mutation. First-line therapies, including pegylated interferon- $\alpha$ and vemurafenib, were able to correct most of these alterations. This study reveals a profound disturbance in the systemic immune phenotype in patients with ECD, providing important new information, helping to understand the physiopathological mechanisms involved in this rare disease and improving the therapeutic management of patients.
\end{abstract}

\section{Introduction}

Erdheim-Chester disease (ECD) is a rare, systemic, nonLangerhans cell histiocytosis neoplasm, frequently caused by mutations in the MEK-extracellular signal-regulated kinase (ERK) signaling pathway; these are mostly BRAF mutations. $E C D$ is characterized by the infiltration of tissues by foamy histiocytes expressing markers of the monocyte/macrophage lineage, including CD45, CD68, CD163, and CD14, whereas ECD histiocytes are negative for CD1a and CD207 dendritic cell (DC) markers. It is proposed that in ECD, histiocytes originate from myeloid $\mathrm{CD}_{3} 4^{+}$and
$\mathrm{CD}_{14}{ }^{+}$progenitor cells. ${ }^{2,3}$ The $B R A F^{\mathrm{V} 600 \mathrm{E}}$ mutation has been detected in hematopoietic stem cells, including common myeloid progenitors and granulocyte-macrophage progenitors, in the bone marrow of patients with $E C D,{ }^{2}$ supporting a model in which BRAF-mutated myeloid cells disseminate from bone marrow to the periphery for tissue infiltration. Consistent with this model, the BRAF ${ }^{\mathrm{V} 600 \mathrm{E}} \mathrm{mu}-$ tation was also found in circulating leukocytes, including classical (CD14 $)$ and nonclassical (CD16 ${ }^{+}$) monocytes and CD1C ${ }^{+}$myeloid DC in individuals with ECD. ${ }^{2}$

The accumulation of histiocytes within lesions in cases of ECD is accompanied by the expression of a chemokine 
and cytokine network favoring immune cell recruitment. ${ }^{4,5}$ Indeed, pro-inflammatory cytokines are highly expressed in ECD lesions, together with the infiltration of pro-inflammatory T-cell helper 1 (Th-1) lymphocytes. In addition, immunohistological examination of ECD biopsies revealed that infiltrated histiocytes express a large set of chemokines and chemokine receptors. ${ }^{4}$ Consistent with these observations, patients with ECD exhibited a systemic immune Th1-oriented cytokine profile, ${ }^{6}$ thereby providing important clues for the therapeutic management of these patients. However, the therapeutic management of patients with ECD remains difficult. First-line therapies are mostly determined by the severity of the disease. Thus, pegylated interferon- $\alpha$ (peg|FN $\alpha$ ) is used to treat mild disease and non-refractory ECD, ${ }^{7}$ whereas drugs targeting the mutated BRAF, such as vemurafenib, are used in multisystemic and refractory ECD. ${ }^{8}$

The underlying mechanisms that orchestrate the immune response in ECD remain largely unknown, and a comprehensive characterization of systemic immune cells in ECD patients is lacking. Therefore, the goal of our study was to determine whether patients with ECD exhibit abnormalities in their systemic immune phenotype and whether these are affected by the presence of the BRAF mutation and therapeutic agents. We demonstrated that patients with ECD had a profound alteration in their systemic immune cell phenotype, characterized by a low abundance of DC subsets and by specific lymphocyte populations, together with a switch in immunoglobulin (Ig) G subclasses, which may be partially corrected by first-line therapies.

\section{Methods}

\section{Patients}

Fasting blood samples were obtained from 17 healthy individuals who formed the control group (13 males and $4 \mathrm{fe}-$ males; mean age; 53 \pm 25 years, range, 21-90 years) and 78 patients with ECD (60 males and 18 females; mean age; $60 \pm 14$ years, range, 18-84 years) who were followed at the Pitié-Salpêtrière Hospital, Paris, France, between December 2012 and July 2015 (Online Supplementary Table S1). For all patients, ECD was diagnosed based on the consensus guidelines for the diagnosis and clinical management of ECD. ${ }^{9}$ The $B R A F^{\mathrm{V} 600 \mathrm{E}}$ mutation was detected using multiplex picodroplet digital polymerase chain reaction analysis (Raindance Technologies), as previously described..$^{10}$ The prevalence of the $B R A F^{\mathrm{V} 600 \mathrm{E}}$ mutation was $64 \%$ in the ECD group (50/71 patients, indeterminate for 7 patients). The absence of the $B R A F^{\mathrm{V} 600 \mathrm{E}}$ mutation in ECD patients was referred to as the wild-type (WT) in this study. At the time of the blood sample collection, the patients were free of any treatment $(n=42)$ or were receiving treatment with either
pegIFNa $(n=31)$, vemurafenib $(n=13)$, or other drugs $(n=17)$. It is worth noting that we cannot determine which of the cases reported in the present study were previously reported. Blood samples were collected from 25 patients at several time points (free of any treatment and upon treatment). This study was approved by the ethics committee of Ile de France III (\#2011-A00447-34) and was conducted in accordance with the Declaration of Helsinki. Informed consent was obtained from all patients.

\section{Analysis of blood immune cells by flow cytometry}

Fresh blood samples were collected in EDTA tubes at the same time of the day for all patients and control individuals; the samples were used immediately for the flow cytometry analysis. Analysis of blood immune cells was carried out simultaneously in both patients and control individuals throughout the study (2013-2015). Similar blood immune cell counts were obtained when flow cytometry analysis was performed for the same control individual at different times of the study. Quantification of the immune cell subsets is described in detail in the Online Supplementary Materials.

\section{Circulating chemokines, cytokines and immunoglobulins}

The quantification of circulating chemokines, cytokines and immunoglobulins is described in the Online Supplementary Materials.

\section{Statistical analyses}

Values are given as medians and interquartile ranges (Q1Q3). Comparisons of two groups were performed using the Mann-Whitney test. Comparisons of more than two groups were performed using the Kruskal-Wallis test followed by a Dunn comparison test. The impacts of the BRAF ${ }^{\mathrm{V} 600 \mathrm{E}} \mathrm{mu}-$ tation and treatment with first-line therapies on blood leukocyte counts were tested using the Jonckheere-Terpstra trend test. Correlations were calculated using the Spearman rank-order test. A $\chi^{2}$ test was performed to analyze the distribution of individuals with ECD around the median value of the indicated parameter, according to BRAF status. For skewed variables, the raw data were logarithmically transformed prior to conducting the analyses. Statistical analyses were performed using $\mathrm{R}$ statistical software version 3.3.2 ( $R$ foundation for Statistical Computing) and Prism software from GraphPad (San Diego, CA USA). Principal component analysis was performed using the public MetaboAnalyst web server (https://wwW.metaboanalyst.ca/).

\section{Results}

Profound alteration of the systemic immune cell phenotype in patients with Erdheim-Chester disease Flow cytometry analysis of blood immune cells in patients 
with ECD allowed the identification of the complete set of monocytes (classical, intermediate, and nonclassical), DC (plasmacytoid [pDC], myeloid 1 [mDC1], and myeloid 2 [mDC2]), and lymphocytes (T-helper [Th], cytotoxic [CT], T regulatory [Treg], natural killer [NK], and $\mathrm{B}$ ) independently of the $B R A F^{\mathrm{V} 600 \mathrm{E}}$ mutation as observed in control individuals (Online Supplementary Figure S1). As shown in Online Supplementary Figure S1, no atypical population was detected in ECD patients, in comparison with control individuals, irrespective of their BRAF status. Although the number of total blood monocytes was higher in patients with ECD who had the BRAF-mutation than in the controls $(+58.9 \%$, $P<0.05)$, none of the monocyte subsets was found to be significantly increased in those individuals (Table 1). Instead, a trend for a decrease in nonclassical CD14 ${ }^{+} \mathrm{CD} 16^{++}$ monocytes was observed in ECD patients carrying the $B R A F^{\mathrm{V} 600 \mathrm{E}}$ mutation $(-73.8 \%, P<0.08)$. More strikingly, a marked decrease in the absolute count of DC, 1349including $\mathrm{PDC}(-63.6 \%, P<0.0005), \mathrm{mDC} 1(-62.0 \%, P<0.05)$, and mDC2 $(-72.6 \%, P<0.005)$, was observed in patients with ECD when compared with the values in healthy individuals; this effect mostly reflected the reduction in all DC subsets in patients with the BRAF-mutation. Such effects were independent of a patient's sex (data not shown). Although the number of blood neutrophils, NK, NKT, and Treg cells was not altered in patients with ECD, we noticed a large decrease in CT $(-80.8 \%, P<0.0005)$ and $\mathrm{B}(-66.5 \%, P<0.005)$ lymphocytes in ECD patients relative to the counts for these cells in controls. Finally, a substantial reduction in the absolute count of Th lymphocytes $(-84.5 \%, P<0.05)$ was observed in patients with ECD who lacked the BRAF mutation.

Principal component analysis of the blood immune cell populations of individuals in the control and ECD groups illustrated the peculiar systemic immune signature that characterized ECD (Figure 1A), as well as a potential effect of the $B R A F^{\mathrm{V} 600 \mathrm{E}}$ mutation, as was suggested by the analysis of individual cell populations (Table 1). Assessment of the impact of the $B R A F^{\mathrm{V} 600 \mathrm{E}}$ mutation on populations of blood immune cells in ECD supports an enhancing effect of the mutation on the reduction in blood nonclassical $\mathrm{CD}_{14}{ }^{+} \mathrm{CD} 16^{++}$monocyte $(P<0.03)$ and $\mathrm{DC}(\mathrm{pDC}, P<0.0002$; $\mathrm{mDC} 1, P<0.05$, and $\mathrm{mDC} 2, P<0.0009)$ numbers and on the increase in blood total monocytes $(P<0.04)$ in ECD patients compared with control individuals (Figure 1B-I).

Analysis of the effect of first-line therapies on this disturbed systemic immune cell signature indicated that patients with ECD who also had the BRAF mutation and who were treated with first-line therapies, including pegIFN $\alpha$ and vemurafenib, did not exhibit such a massive alteration of the systemic immune cell phenotype when compared with control individuals (Table 1). As an illustration, the absolute counts of $\mathrm{mDC} 1$ and $\mathrm{mDC} 2$ populations in treated ECD patients carrying the BRAF ${ }^{\mathrm{V}}{ }^{00 E}$ mutation were not significantly different from those of control individuals. Assessment of the impact of first-line therapies in ECD patients with the BRAF mutation highlighted the capacity of treatments to partially correct or restore the circulating numbers of several altered leukocyte populations in ECD; this was observed for nonclassical $\mathrm{CD}_{14}{ }^{+} \mathrm{CD} 16^{++}$monocyte $(P<0.03), \mathrm{mDC} 1(P<0.03)$, and $\mathrm{mDC} 2$ $(P<0.0006)$ populations (Figure 2$)$. However, treatments taken individually or as a whole were unable to restore the decrease in PDC populations in patients with ECD (Table 1 and Figure 2).

Taken together, these findings highlight a major perturbation of the systemic immune cell phenotype in ECD cases, characterized by a deficit of DC and lymphocytes, which could be partially restored by first-line treatments in patients with the BRAF mutation.

\section{Impact of first-line therapies on the systemic cytokine and chemokine network in patients with Erdheim- Chester disease}

To provide clues about the mechanism underlying the alteration of the systemic immune cell phenotype of patients with ECD according to the BRAF status, a comprehensive quantification of circulating chemokines and cytokines was performed on this single-center group of 78 patients with ECD (Online Supplementary Table S2). As previously reported, ${ }^{6}$ the levels of many circulating cytokines and chemokines are highly heterogeneous among ECD patients (Online Supplementary Table S2). However, when we investigated the impact of the $B R A F^{\mathrm{V} G 00 \mathrm{E}}$ mutation on ECD patients' cytokine and chemokine profiles, we observed that the proportion of individuals with high levels of numerous circulating cytokines driving the Th1 response (IL-6, IL-8, IL-12p40, and TNF $\alpha$ ) and chemokines (IP-10, CCL2, MIP-1 $\alpha$, and CCL22) was higher in carriers of the $B R A F^{\mathrm{V} G 00 E}$ mutation than in noncarriers (Figure 3). It is noteworthy that patients with the $B R A F-$ mutation also exhibited high levels of the anti-inflammatory cytokine, IL-10. In contrast, a higher proportion of patients with elevated circulating levels of eotaxin, EGF, and IL-15 was detected among patients lacking the $B R A F$ mutation than in their counterparts with the BRAF mutation. Because of the specific mode of action of the treatments, i.e., vemurafenib and pegIFN $\alpha$, no difference in the circulating concentrations of cytokines and chemokines was detected between ECD patients with the BRAF mutation who were treated or not treated with first-line therapies when taken as a whole (Online Supplementary Table S2). Conversely, compared with their non-treated counterparts, ECD patients with the BRAF mutation who were treated with pegIFN $\alpha$ exhibited higher circulating levels of cytokines that drive either a pro-inflammatory Th1 (IFNa, 5.6-fold, $P<0.0001$; and IL-15, 1.8-fold, $P<0.05$ ) or an anti-inflammatory Th2 (IL-10, 1.9-fold, $P<0.05)$ re- 



Figure 1. Patients with Erdheim-Chester disease are characterized by a peculiar systemic immune cell signature (A-I) Principal component analysis (A) and blood counts of total (B) and nonclassical (C) monocytes, T helper (D), cytotoxic (E) and B (F) lymphocytes, and plasmacytoid $(\mathrm{G})$, and myeloid $1(\mathrm{H})$ and $2(\mathrm{I})$ dendritic cells in patients with untreated Erdheim-Chester disease (ECD) according to their BRAF status in comparison with individuals in the control group. Controls, $n=17$; ECD patients without the BRAF mutation (WT), $n=11$; and ECD patients carrying the BRAFV600E mutation (V600E), $n=23$. P-value for the trend was assessed using the Jonckheere-Terpstra trend test. 


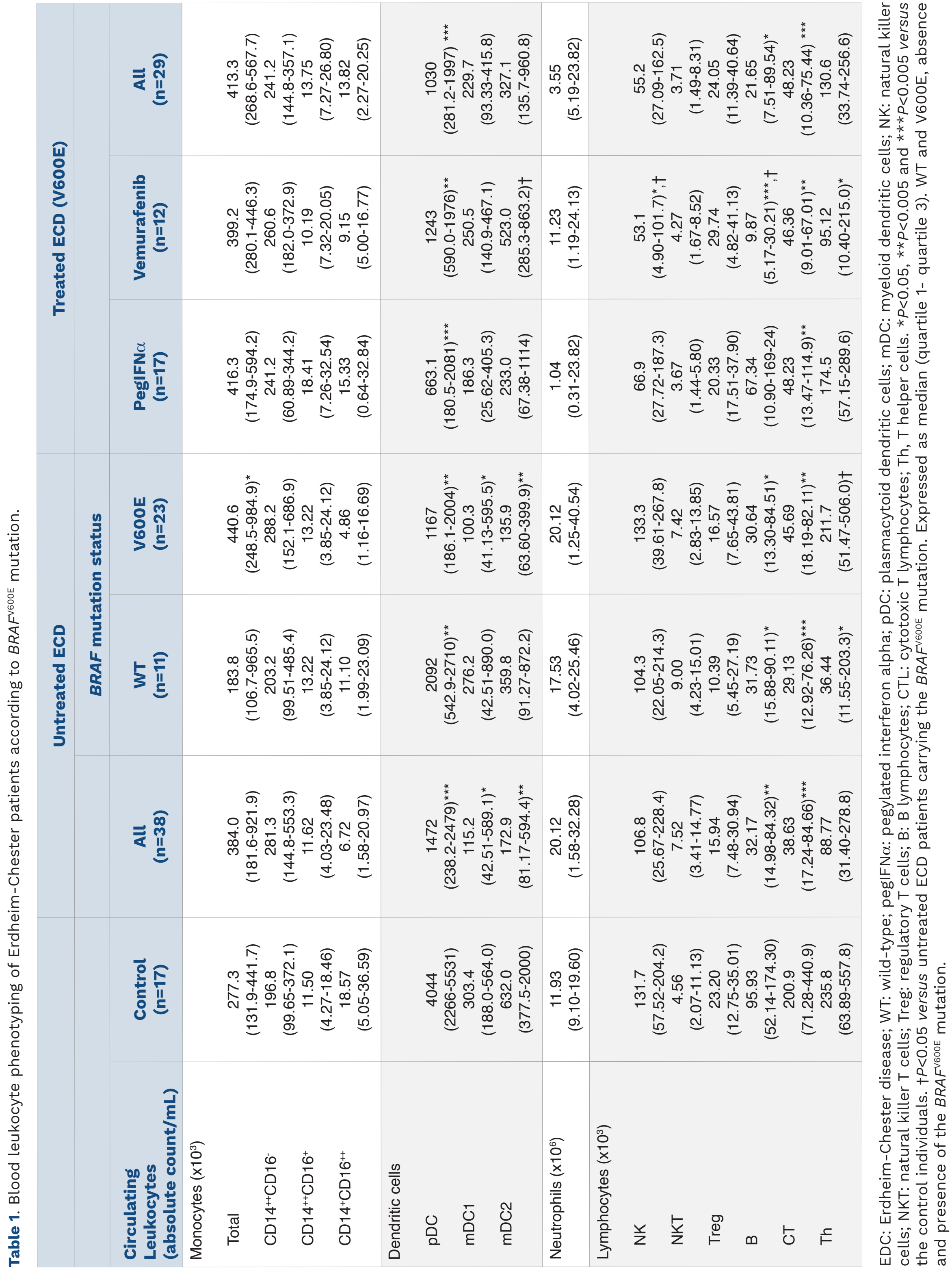


sponse, as well as higher levels of chemokines (IP-10, 1.5fold, $P<0.05$; and CCL2, 1.3-fold, $P<0.05)$ and a cytokine involved in hematopoiesis (GCSF; 2.2-fold, $P<0.05$ ) (Online Supplementary Table S2). However, it is worth mentioning that there was a reduction in plasma CCL22 levels (-34\%, $P<0.05)$ in ECD patients carrying the BRAF ${ }^{\mathrm{V} 600 \mathrm{E}}$ mutation, after they received pegIFN $\alpha$ treatment. A similar pegIFN $\alpha$ signature was observed when all patients with ECD were considered, irrespective of their BRAF status (data not shown). Finally, a significant reduction in plasma CCL2 levels was only observed in ECD patients upon vemurafenib therapy compared with levels in untreated ECD patients carrying the BRAF mutation $(-49.5 \%, P<0.05)$ (Online Supplementary Table S2).

Taken together, our results show that patients with ECD who carried the $B R A F^{\mathrm{V} 600 \mathrm{E}}$ mutation had an overall more

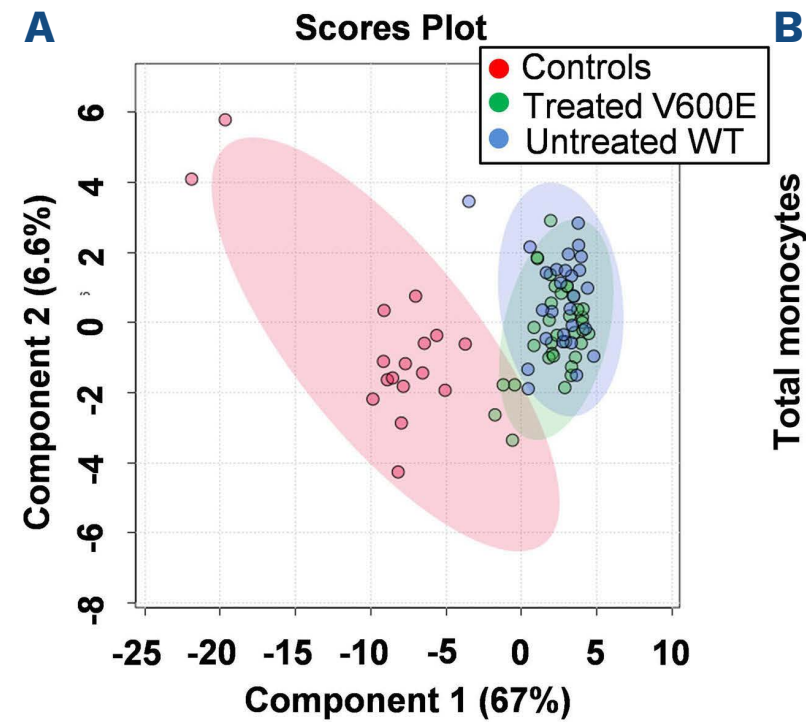

B

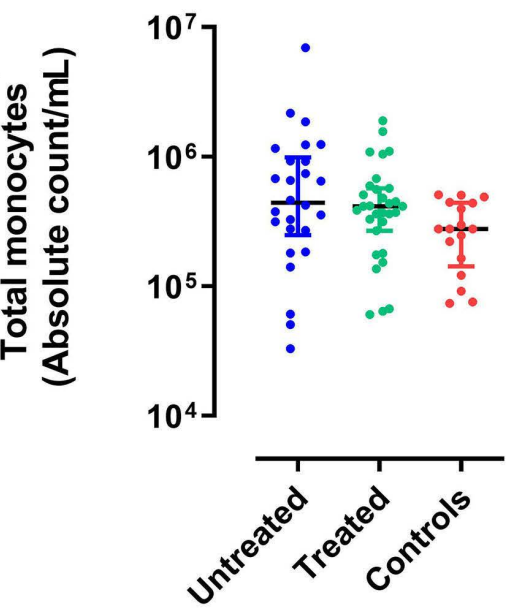

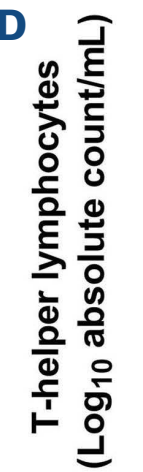
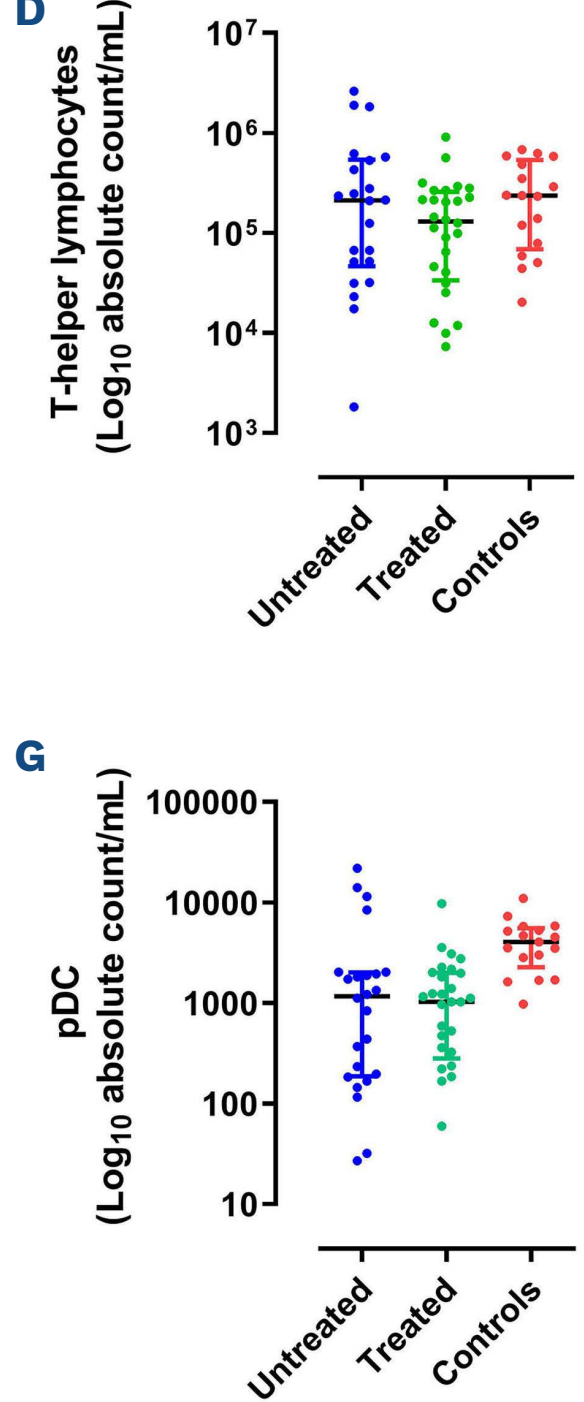
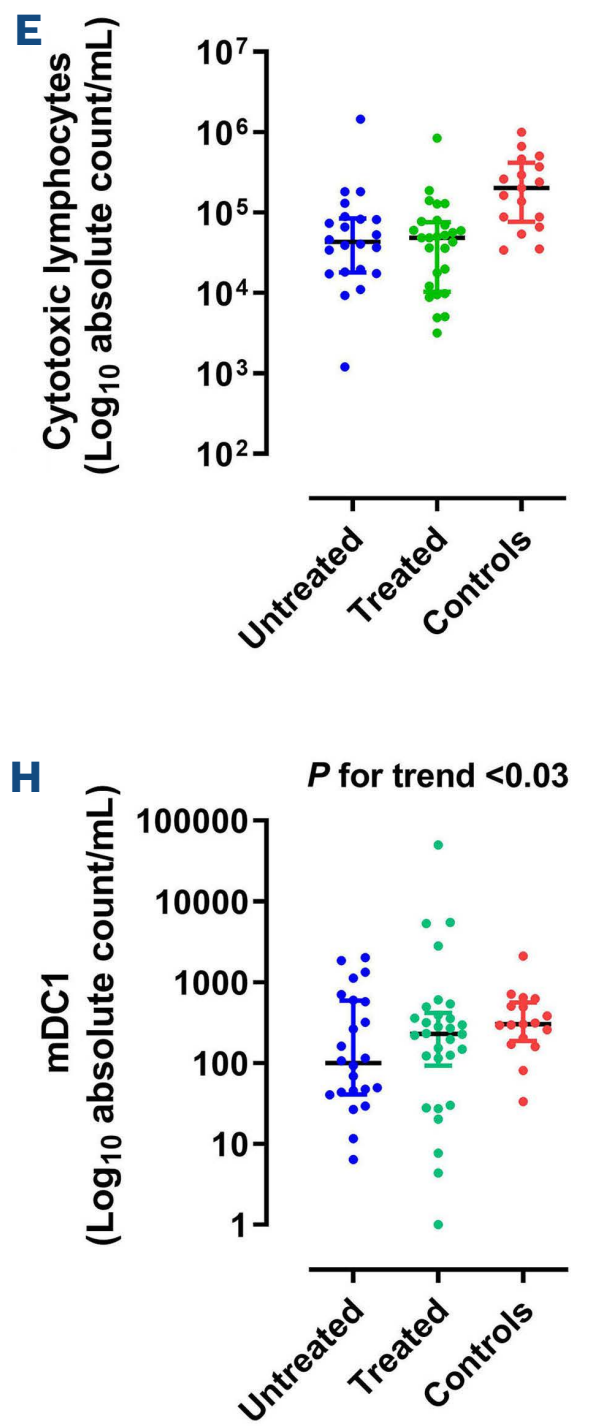
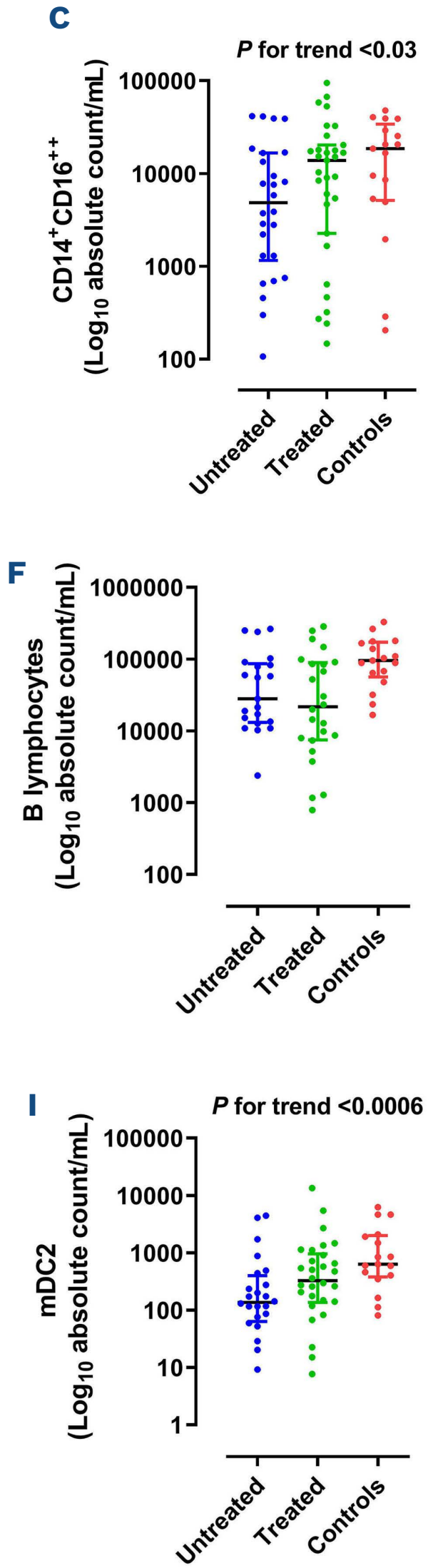

Figure 2. Impact of first-line therapies on the systemic immune cell signature in patients with Erdheim-Chester disease carrying the BRAF mutation. (A-I) Principal component analysis (A) and blood counts of total (B) and nonclassical (C) monocytes, T helper (D), cytotoxic (E) and B (F) lymphocytes, and plasmacytoid (G), and myeloid $1(H)$ and 2 (I) dendritic cells in untreated or treated patients with Erdheim-Chester disease (ECD) carrying the BRAF ${ }^{\mathrm{V} 000 \mathrm{E}}$ mutation in comparison with individuals in the control group. Controls $(n=17)$, untreated $(n=23)$, and treated $(n=29)$ ECD patients carrying the BRAF ${ }^{6000 E}$ mutation (V600E). Treatments included pegylated interferon $\alpha$ and vemurafenib. $P$-value for the trend was assessed using the Jonckheere-Terpstra trend test. 
pro-inflammatory cytokine and chemokine signature than ECD patients who did not carry this mutation, and this signature appears to have been further exacerbated by pegIFN $\alpha$ treatment.

Interrelationship between blood immune cell phenotype and cytokine and chemokine network in patients with

\section{Erdheim-Chester disease}

We next investigated whether the modifications to the circulating cytokine and chemokine concentrations may translate into the major perturbation of the systemic immune cell phenotype, as well as its partial restoration following first-line therapy, in ECD patients carrying the $B R A F$ mutation. For this purpose, correlations were explored between circulating immune cell numbers and the concentrations of cytokines and chemokines, in the entire ECD cohort As shown in Online Supplementary Table S3, although none of these biomolecules was found to be correlated with blood B or Th lymphocyte levels, the results indicated that the absolute count of nonclassical monocytes $\left(\mathrm{CD} 14^{+} \mathrm{CD} 16^{++}\right)$was positively correlated with the plasma concentrations of IFN $22(r=0.31, P<0.005)$, IL$6(r=0.30, P<0.05), I L-8(r=0.23, P<0.05)$, and IL-5 $(r=0.27$, $P<0.05)$. Interestingly, plasma IP-10 levels were positively correlated with the abundance of both nonclassical monocytes $(r=0.31, P<0.05)$ and mDC2 cells $(r=0.28$, $P<0.05)$ in the blood, while a correlation was detected between TNF $\alpha$ and MIP-1 $\beta$ levels with the number of mDC1 cells $(r=0.40, P<0.0005)$ and CT lymphocytes $(r=-0.24$, $P<0.05)$.

As a whole, these findings have led to the identification of a set of cytokines and chemokines that might account for the abundance of nonclassical monocytes and myeloid DC following first-line therapies in the blood of ECD patients who carry the BRAF mutation.
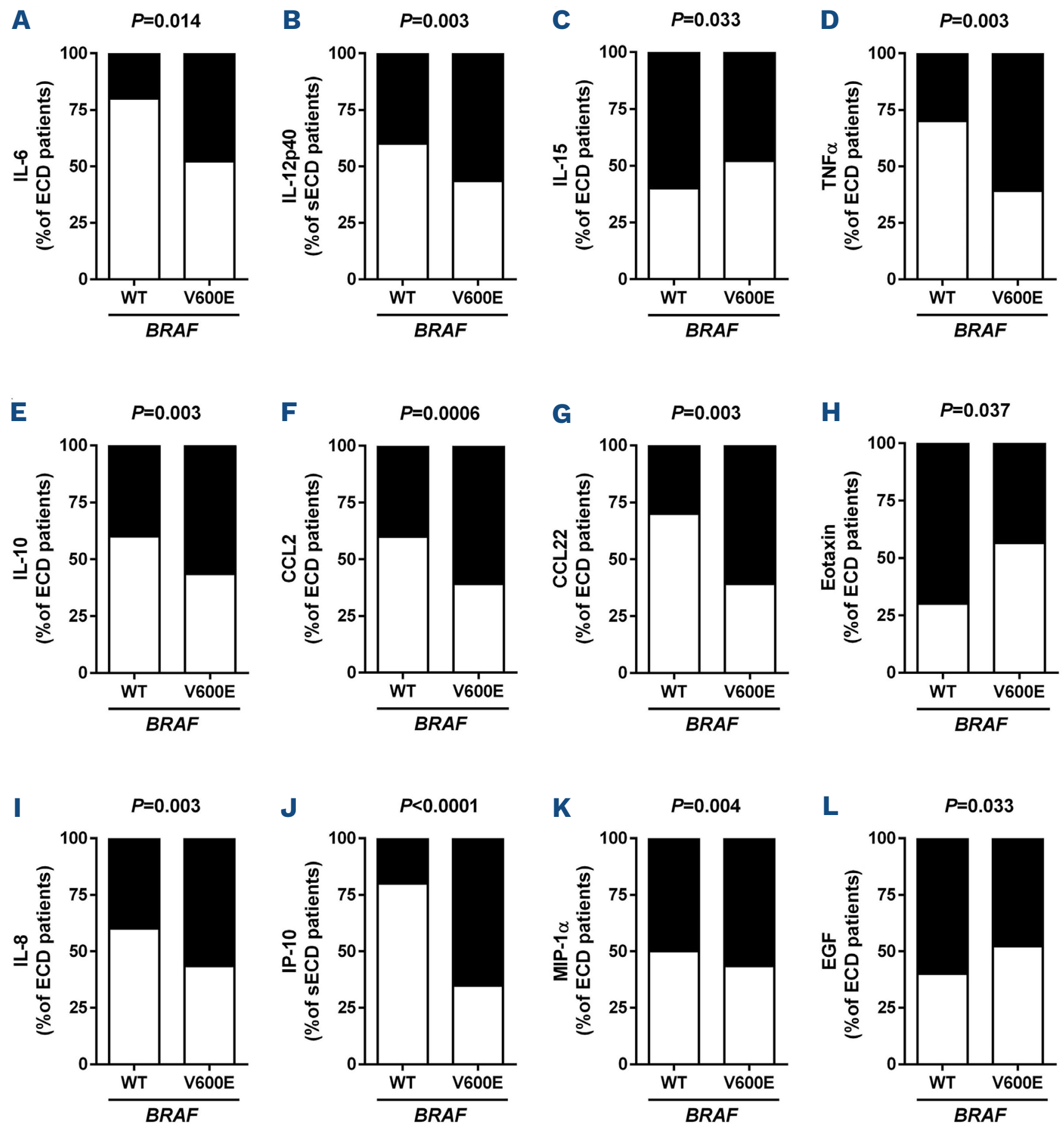

Below median value
Figure 3. Impact of the BRAF ${ }^{\mathrm{v} 600}$ mutation on the systemic chemokine and cytokine network in patients with Erdheim-Chester disease.(A-L) Analysis of the repartition of untreated patients with Erdheim-Chester disease (ECD) according to their BRAF status around the median values of systemic concentrations of IL-6 (A), IL-12p40 (B), IL-15 (C), TNF $\alpha$ (D), IL-10 (E), CCL2 (F), CCL22 $(\mathrm{G})$, eotaxin $(\mathrm{H}), \mathrm{IL}-8(\mathrm{I}), \mathrm{IP}-10(\mathrm{~J})$, MIP-1 $\alpha(\mathrm{K})$, and EGF (L). ECD patients without the BRAF mutation (WT), $\mathrm{n}=9$ and ECD patients carrying the $B R A F^{\mathrm{V} 600 \mathrm{E}}$ mutation (V600E), $\mathrm{n}=21$. Statistical significance was tested using a $\chi^{2}$ test. 
Immunoglobulin switch toward immunoglobulin G4 in patients with Erdheim-Chester disease

Finally, to determine whether the disturbance of the systemic immune cell phenotype translates into a defect in immunoglobulin production, plasma concentrations of immunoglobulin isotypes (IgA, IgM, IgG1, IgG2, IgG3, and IgG4) were quantified in patients with untreated ECD. Although the quantities of IgA and IgM were within the reference ranges for adults, ${ }^{12}$ those of IgG were more elevated, which mostly reflected the high abundance of IgG4 and, to a lesser extent, high concentrations of IgG2 ${ }^{13}$ (Online Supplementary Table S4). As a result, in patients with ECD, the proportion of IgG1 (IgG1/IgGs) was low, whereas that of IgG4 (IgG4/IgGs) was high; this effect appeared to be more pronounced in patients carrying the $B R A F$ mutation. Analysis of the distribution of patients according to their IgG4 levels (normal <135 and high $\geq 135$ $\mathrm{mg} / \mathrm{dL})^{14}$ indicated that whereas a roughly similar proportion of patients without the BRAF mutation had either normal or high levels of IgG4, the level of IgG4 in patients carrying the BRAF ${ }^{\mathrm{V} 600 \mathrm{E}}$ mutation was 1.7 -fold higher than that of patients who lacked the mutation in the normal
IgG4 group and up to 3-fold higher in the high IgG4 group (Figure 4A). Overall, $64.5 \%$ of patients with ECD had a high IgG4 immune phenotype and this was predominantly among those patients who carried the BRAF mutation. Strikingly, first-line therapies corrected the IgG switch in this latter group, with a significant increase in IgG1 being observed upon pegIFN $\alpha$ therapy (untreated, 30.9 [25.343.8] versus pegIFN $\alpha, 50.3$ [36.1-58.3], $P<0.005)$, while IgG4 returned to normal values following vemurafenib treatment (untreated, 16.5 [6.51-37.4] versus vemurafenib, 4.69 [2.02-7.94], $P<0.05$ ) (Figure 4). A similar correction of the IgG profile was also observed for the entire ECD cohort treated with first-line therapies (Online Supplementary Figure S2). These findings revealed that patients with ECD exhibited an IgG switch, from IgG1 to IgG4, which was corrected by first-line therapies.

\section{Discussion}

The present study, involving a single-center series of 78 patients with ECD, revealed a profound perturbation of
A

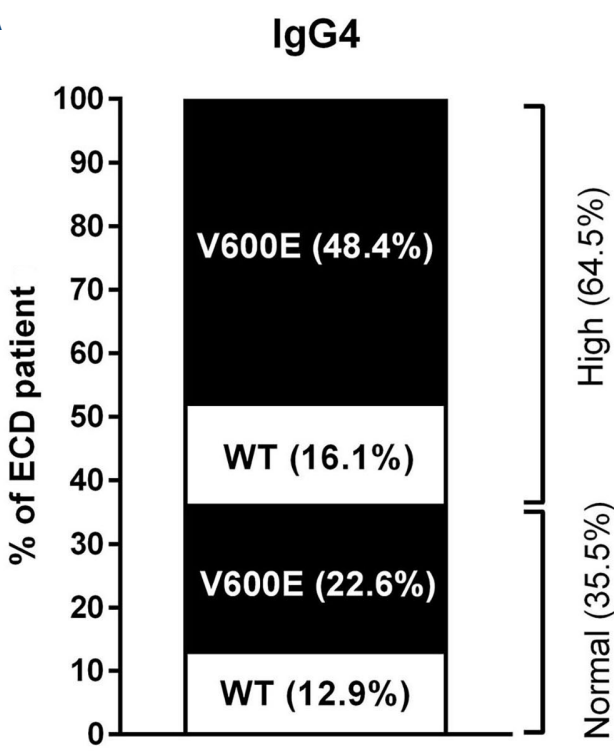

D

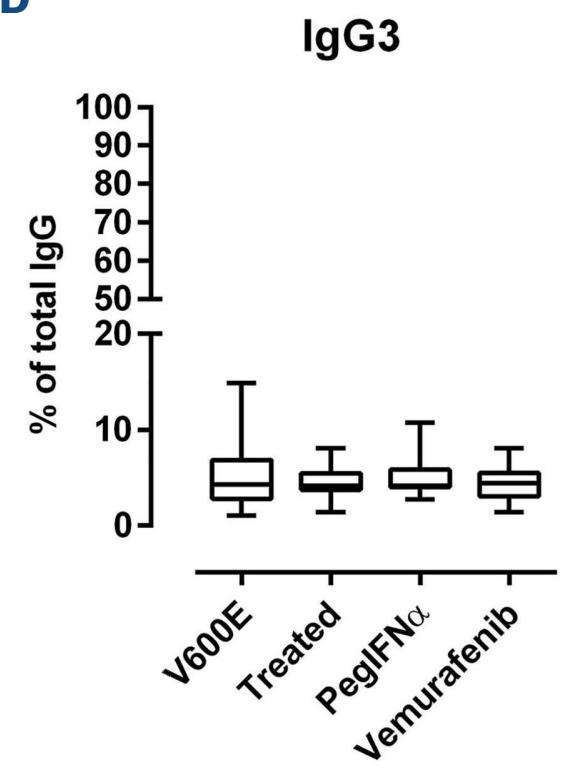

B

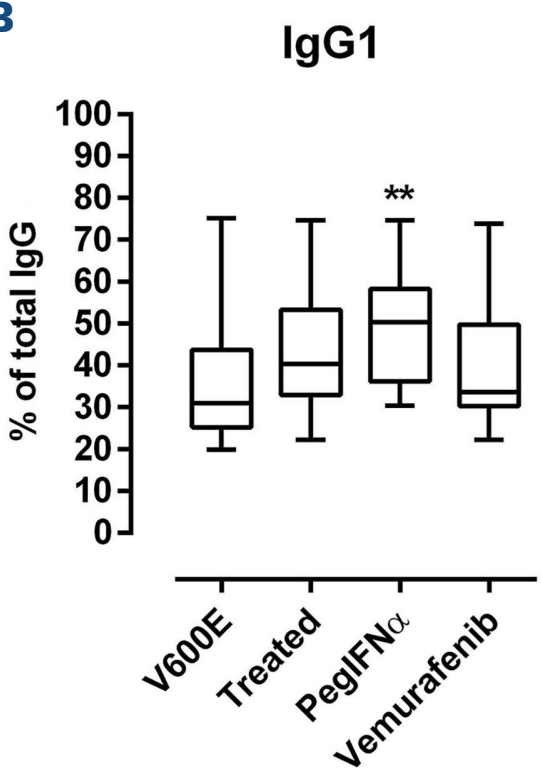

$\mathbf{E}$

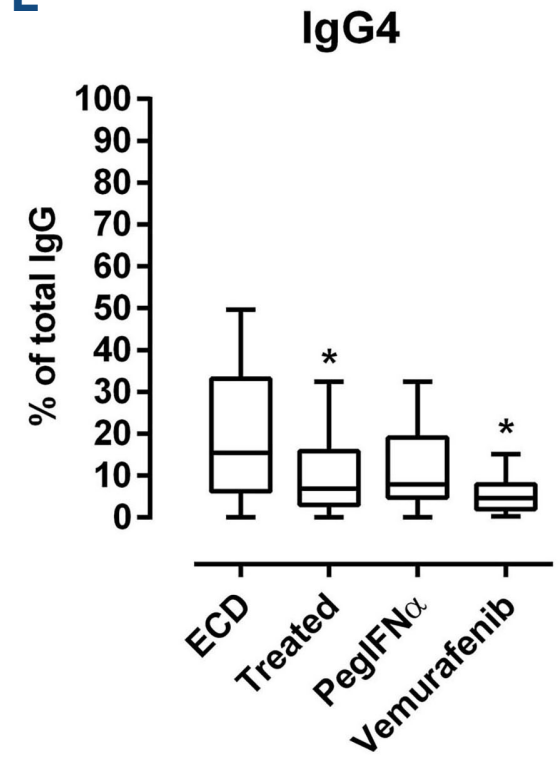

C

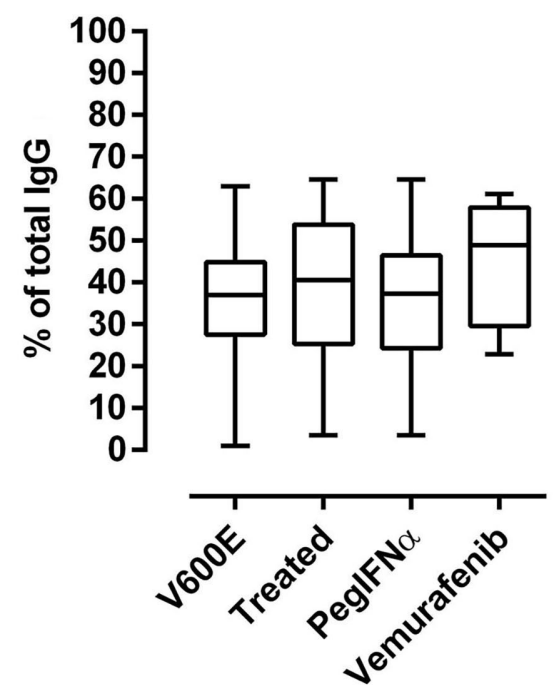

Figure 4. Correction of the IgG1/IgG4 switch by first-line therapies in patients carrying the $B R A F$ mutation. (A) Prevalence of the high-level IgG4 phenotype in untreated patients with ErdheimChester disease (ECD) according to the presence of the $B R A F^{\mathrm{V} 600 \mathrm{E}}$ mutation. Normal IgG4 $<135$ $\mathrm{mg} / \mathrm{dL}$, high IgG4 $\geq 135 \mathrm{mg} / \mathrm{dL}$. ECD patients without the BRAF mutation (WT), $\mathrm{n}=9$ and $\mathrm{ECD}$ patients carrying the $B R A F^{\mathrm{V} 600 \mathrm{E}}$ mutation (V600E), $n=22$. (B-E) Impact of first-line therapies on the percentage of IgG1 (B), IgG2 (C), IgG3 (D), and IgG4 (E). Untreated $(n=22)$ and treated $(n=27$; peg|FN $\alpha=16$ and vemurafenib=11) ECD patients carrying the BRAF ${ }^{\mathrm{V} 600 \mathrm{E}}$ mutation (V600E). Differences between groups were tested using the Kruskal-Wallis test. $* P<0.05$ and $* * P<0.005$ versus untreated $E C D$ patients carrying the $B R A F^{\mathrm{V} 600 \mathrm{E}}$ mutation. 
the blood immune phenotype in these patients, characterized by a decrease in the DC and lymphocyte populations accompanied by a switch in IgG subclasses. This perturbation was exacerbated in patients carrying the $B R A F^{\mathrm{V} 600 \mathrm{E}}$ mutation, who also exhibited a higher pro-inflammatory status than patients who lacked this mutation. First-line therapies were able to partially correct the altered immune cell phenotype and restore the IgG pattern.

This first comprehensive analysis of systemic immune cell populations in patients with ECD revealed a peculiar immune cell ECD signature, characterized by a very low abundance of $\mathrm{DC}$, including $\mathrm{PDC}, \mathrm{mDC} 1$, and $\mathrm{mDC} 2$, in comparison with the abundance of these cells in matched control individuals. Although there is limited information available about the levels of immune cells in the blood of patients with histiocytosis, this observation contrasts with the increased quantity of DC precursors detected in the blood of patients with Langerhans cell histiocytosis, a histiocytic neoplasm that arises from the dendritic lineage..$^{15}$ Although a trend for such a decrease in DC was observed in patients who lacked the BRAF mutation, a much stronger effect was detected in patients who did carry this mutation, suggesting that the activation of the ERK signaling pathway could underlie this phenotype. The perturbation in blood DC levels was unlikely to have resulted from the increased infiltration of these cells into tissues, as no $\mathrm{CD} 123^{+}$cells (PDC) have previously been detected in ECD lesions. ${ }^{6}$ Rather, activation of the MEK/ERK signaling pathway was reported to inhibit the maturation of monocyte-derived DC. ${ }^{16,17}$ More recently, Hogstad et al. elegantly demonstrated that the MAPK pathway, including the $B R A F^{V 600 E}$ mutation, suppresses DC migration and traps DC in Langerhans cell histiocytosis lesions. ${ }^{18}$ BRAF mutations have been detected in myeloid progenitors in bone marrow from ECD patients; ${ }^{2}$ therefore, our findings lead us to propose that the presence of the $B R A F^{\mathrm{V}}{ }^{600} \mathrm{E}$ mutation in myeloid DC precursors might cause these cells to be retained in the bone marrow compartment and impede their migration to the blood circulation. This mechanism could explain the paradoxical elevated systemic IFN $\alpha$ concentrations reported in patients with ECD, ${ }^{6}$ despite the low abundance of blood DC described here. Additional investigations are needed to determine whether an increase in myeloid DC precursors can be detected in the bone marrow of patients with ECD.

Antigen-presenting cells such as DC interact with lymphocytes and contribute to their proliferation and maturation and the establishment of an immune response. Together with the decrease in blood DC, the systemic concentrations of helper, cytotoxic, and B lymphocytes were markedly reduced in patients with ECD in the present study. Moreover, decreased systemic levels of IL-7, a cytokine involved in B- and T-lymphocyte differentiation, have been reported in patients with ECD. ${ }^{6}$ The infiltration of Th1 cells into ECD lesions ${ }^{4}$ could also contribute to the reduction in the abundance of circulating $T$ lymphocytes. Indeed, CCL19/MIP-3 $\beta$, a chemo-attractant for B and T lymphocytes and DC, was reported to be expressed in ECD lesions that were analyzed by immunohistochemistry. ${ }^{4}$ In contrast, the expansion of Treg lymphocytes alone in both the blood compartment and lesions has been reported in Langerhans cell histiocytosis, while monocyte and DC populations were not altered. ${ }^{19}$ Despite the low abundance of circulating B cells, a recent study pointed out the high prevalence $(42 \%)$ of autoimmunity in patients with ECD. ${ }^{20}$ Here, we brought to light perturbations in the IgG profile characterized by high IgG4 levels and leading to an IgG1/IgG4 switch. A few case reports have documented high IgG4 levels in ECD patients, suggesting ECD mimics IgG4-related disease. ${ }^{14,21}$ In a review of a single-center cohort, Gianfreda et al. observed that high levels of IgG4 were present in $26.7 \%$ (4/15) of patients with ECD. ${ }^{14}$ In the present study, involving 78 patients, high levels of IgG4 ( $\geq 135 \mathrm{mg} / \mathrm{dL}$ ) were observed more frequently, affecting $64.5 \%$ of patients. However, while ECD and IgG4-related disease share some physiopathological characteristics, these diseases have distinctive clinical features, suggesting they are distinct disorders. The increased production of IgG4 is frequently driven through a Th2 response to IL-4, IL-5, or IL-13 and by anti-inflammatory IL-10 and TGF $\beta$ cytokines. ${ }^{22}$ Although ECD patients exhibit a Th1 immune response, ${ }^{4,6}$ the present study indicated that patients who carry the BRAF mutation have higher circulating IL-10 concentrations and are more likely to exhibit high IgG4 levels than patients who lack the mutation, suggesting that IL-10 might contribute to the IgG4 immune response in ECD. Moreover, IFN $\alpha$, which is secreted by PDC and initiates the Th1 response and whose systemic concentrations are elevated in ECD, ${ }^{6}$ has been reported to increase IgG4 production by $B$ lymphocytes. ${ }^{23}$ Thus, infiltration of pDC in pancreatic lesions of patients with IgG4-related autoimmune pancreatitis has been proposed to induce IgG4 production by plasma cells via IFNa..$^{23}$ Similar to what is observed in cases of IgG4-related disease, IgG4-positive plasma cell infiltrates were observed in ECD lesions at perirenal and subcutaneous sites. ${ }^{14}$ Whether or not the reduction in circulating $B$ cells in ECD detected in the present study reflects the infiltration of these cells into lesions or impaired B cell differentiation deserves further investigation.

The quantification of serum cytokines in a single-center series of 37 patients with ECD was previously reported; it included the identification of an ECD signature based on the concentrations of IFN 22 , IL-12, MCP-1, IL-4, and IL-7, which allowed ECD patients to be distinguished from control individuals. ${ }^{6}$ The present study provides new information regarding the effect of the $B R A F^{\mathrm{V}}{ }^{600} \mathrm{E}$ mutation on this 
ECD signature, as well as on the systemic immune Th1 phenotype that characterizes ECD. We found an exacerbated Th1-mediated systemic immune response in patients carrying the BRAF mutation, characterized by a higher proportion of participants carrying the $B R A F^{\mathrm{V} 600 \mathrm{E}}$ mutation with elevated circulating concentrations of proinflammatory cytokines (IL-12p40, IL-6, IL-8, and TNF $\alpha$ ) and chemokines (IP-10, CCL2, CCL22, and MIP-1 $\alpha$ ) than participants who lacked this mutation. However, first-line therapies were unable to dampen this pro-inflammatory phenotype. Elevated levels of circulating chemokines in patients carrying the BRAF mutation are consistent with those in previous studies that found that the presence of the $B R A F^{\mathrm{V} 600 \mathrm{E}}$ mutation is a major determinant in histiocyte infiltration ${ }^{24}$ and that vemurafenib shows a high efficacy in multisystemic and refractory ECD. ${ }^{8}$ The decrease in CCL2 concentrations in ECD patients treated with vemurafenib in comparison with their untreated counterparts carrying the BRAF mutation might account, at least in part, for the reduced infiltration upon receiving this therapy. An analysis of the systemic chemokine and cytokine network in 52 patients with Langerhans cell histiocytosis versus 34 control individuals revealed that patients carrying the $B R A F^{\mathrm{V} 600 \mathrm{E}}$ mutation only showed elevated levels of MCP-3 in serum, with no other abnormalities detected. ${ }^{25}$ Such an elevation of MCP-3 was not observed in patients carrying the BRAF mutation in the present study.

In the present study, we found that first-line therapies, although having a modest impact on the systemic chemokine and cytokine concentrations, were able to correct most of the alterations in blood immune cell counts, especially those of nonclassical $C D 14^{+} \mathrm{CD} 16^{++}$monocytes,
mDC1, mDC2, and B lymphocytes, whereas they failed to restore those of pDC. While disturbances in the immune response were more frequent in ECD patients carrying the $B R A F$ mutation, targeted therapy appeared to be less effective than pegIFN $\alpha$ in these patients. Nevertheless, vemurafenib, similarly to pegIFN $\alpha$, corrected the IgG1/IgG4 switch. The major findings of the present study are summarized in Figure 5.

The limitations of this study include the relatively low number of patients with ECD on the different therapies (vemurafenib versus pegIFN $\alpha$ ). Another limitation is the absence of data from ECD patients before and after treatment, which would be useful to investigate in more detail the impact of first-line therapies on the systemic disturbance of the immune cell phenotype and the IgG switch. Finally, the inclusion of a control group comprising patients with Langerhans cell histiocytosis would have helped as a comparison with the specific inflammatory patterns in ECD.

In conclusion, our study is the first to document the marked alteration of the systemic immune response in ECD and brings to light the involvement of DC in this non- Langerhans cell histiocytosis neoplasm. This new information will help our understanding of the mechanisms taking place in ECD physiopathology and provides additional clues to the best approach to the therapeutic management of patients with ECD.

\section{Disclosures}

No conflicts of interest to disclose.

\section{Contributions}

FCA, LP, FS-C, YA, EF, and WLG performed the research.

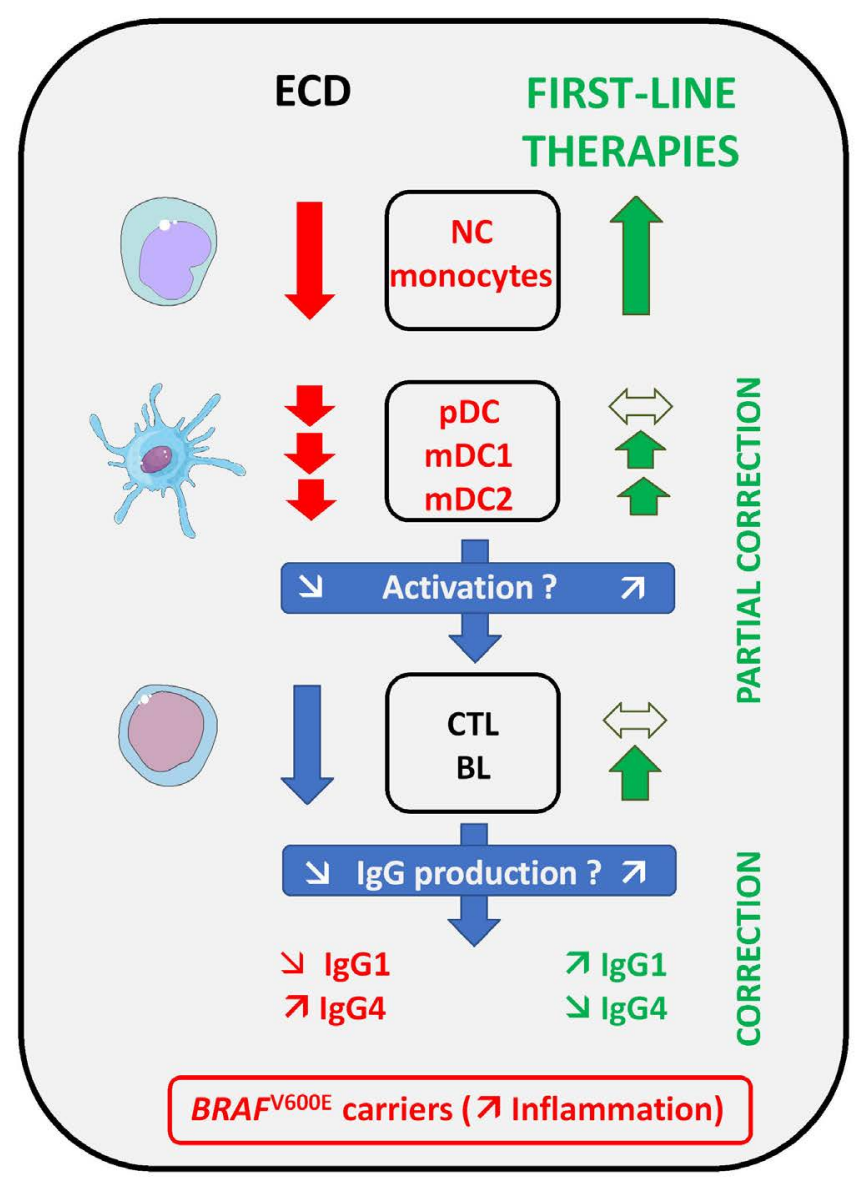

Figure 5. Major alterations of the systemic immune cell phenotype in patients with Erdheim-Chester disease. Flow cytometry analysis of blood leukocytes in patients with Erdheim-Chester disease revealed a marked decrease in dendritic cells ( $\mathrm{pDC}, \mathrm{mDC1}$, and mDC2) and lymphocytes (CTL and $\mathrm{BL}$ ), as well as a reduction in nonclassical monocytes in comparison with levels of these cells in individuals in the control group. Such a reduction in antigen-presenting cells might impair the activation of cytotoxic $T$ lymphocytes and $B$ lymphocytes and the production of IgG, leading to an IgG switch toward IgG4. These alterations were mostly observed in ECD patients carrying the BRAF ${ }^{\mathrm{V} 600 \mathrm{E}}$ mutation (in red), who exhibited a more pronounced systemic inflammation. First-line therapies partially corrected the systemic immune cell phenotype and normalized blood IgG concentrations. BL: B lymphocytes; CTL: cytotoxic T lymphocytes; IgG: immunoglobulin G; NC: nonclassical; mDC: myeloid dendritic cells; pDC: plasmacytoid dendritic cells. 
FCA, PL, JH, and WLG designed the research. FCA and JH recruited the patients. MG and WLG analyzed the data. $J H$ and WLG obtained funding for the research. All authors gave critical comments on the manuscript. WLG supervised the study and wrote the manuscript.

\section{Acknowledgments}

The authors are indebted to all the participants for their cooperation.

\section{Funding}

INSERM, Sorbonne Université, French National Agency (ANR-10-IAHU-05), and the Erdheim-Chester Disease Global Alliance.

\section{References}

1. Durham BH. Molecular characterization of the histiocytoses: neoplasia of dendritic cells and macrophages. Semin Cell Dev Biol. 2019;86:62-76.

2. Milne P, Bigley $V$, Bacon $C M$, et al. Hematopoietic origin of Langerhans cell histiocytosis and Erdheim-Chester disease in adults. Blood. 2017;130(2):167-175.

3. Durham BH, Roos-Weil D, Baillou C, et al. Functional evidence for derivation of systemic histiocytic neoplasms from hematopoietic stem/progenitor cells. Blood. 2017;130(2):176-180.

4. Stoppacciaro A, Ferrarini M, Salmaggi C, et al. Immunohistochemical evidence of a cytokine and chemokine network in three patients with Erdheim-Chester disease: implications for pathogenesis. Arthritis Rheum. 2006;54(12):4018-4022.

5. Dagna L, Girlanda S, Langheim S, et al. Erdheim-Chester disease: report on a case and new insights on its immunopathogenesis. Rheumatology. 2010;49(6):1203-1206.

6. Arnaud L, Gorochov G, Charlotte F, et al. Systemic perturbation of cytokine and chemokine networks in Erdheim-Chester disease: a single-center series of 37 patients. Blood. 2011;117(10):2783-2790.

7. Cohen-Aubart F, Maksud P, Emile J-F, et al. Efficacy of infliximab in the treatment of Erdheim-Chester disease. Ann Rheum Dis. 2018;77(9):1387-1390.

8. Haroche J, Cohen-Aubart F, Emile J-F, et al. Dramatic efficacy of vemurafenib in both multisystemic and refractory ErdheimChester disease and Langerhans cell histiocytosis harboring the BRAF V600E mutation. Blood. 2013;121(9):1495-1500.

9. Goyal G, Heaney ML, Collin M, et al. Erdheim-Chester disease: consensus recommendations for evaluation, diagnosis, and treatment in the molecular era. Blood. 2020;135(22):1929-1945.

10. Haroche J, Charlotte F, Arnaud L, et al. High prevalence of BRAF V600E mutations in Erdheim-Chester disease but not in other non-Langerhans cell histiocytoses. Blood. 2012;120(13):27002703.

11. Szalat R, Pirault J, Fermand J-P, et al. Physiopathology of necrobiotic xanthogranuloma with monoclonal gammopathy. $J$ Intern Med. 2014;276(3):269-284.

12. Gonzalez-Quintela A, Alende R, Gude F, et al. Serum levels of immunoglobulins (IgG, IgA, IgM) in a general adult population and their relationship with alcohol consumption, smoking and common metabolic abnormalities: serum immunoglobulin levels in adults. Clin Exp Immunol. 2008;151(1):42-50.

13. Schauer $\mathrm{U}$, Stemberg $\mathrm{F}$, Rieger $\mathrm{CHL}$, et al. IgG subclass concentrations in certified reference material 470 and reference values for children and adults determined with the binding site reagents. Clin Chem. 2003;49(11):1924-1929.

14. Gianfreda D, Musetti C, Nicastro M, et al. Erdheim-Chester disease as a mimic of IgG4-related disease: a case report and a review of a single-center cohort. Medicine (Baltimore). 2016;95(21):e3625.

15. Rolland A, Guyon L, Gill M, et al. Increased blood myeloid dendritic cells and dendritic cell-poietins in Langerhans cell histiocytosis. J Immunol. 2005;174(5):3067-3071.

16. Puig-Kröger A, Relloso M, Fernández-Capetillo $O$, et al. Extracellular signal-regulated protein kinase signaling pathway negatively regulates the phenotypic and functional maturation of monocyte-derived human dendritic cells. Blood. 2001;98(7):2175-2182.

17. Aguilera-Montilla N, Chamorro S, Nieto C, et al. Aryl hydrocarbon receptor contributes to the MEK/ERK-dependent maintenance of the immature state of human dendritic cells. Blood. 2013;121(15):e108-117.

18. Hogstad B, Berres M-L, Chakraborty R, et al. RAF/MEK/extracellular signal-related kinase pathway suppresses dendritic cell migration and traps dendritic cells in Langerhans cell histiocytosis lesions. J Exp Med. 2018;215(1):319-336.

19. Senechal B, Elain G, Jeziorski E, et al. Expansion of regulatory $T$ cells in patients with Langerhans cell histiocytosis. PLoS Med. 2007;4(8):e253.

20. Roeser A, Cohen-Aubart F, Breillat P, et al. Autoimmunity associated with Erdheim-Chester disease improves with BRAF/MEK inhibitors. Haematologica. 2019;104(11):e502-e505.

21. Miron G, Karni A, Faust-Soher A, Giladi N, Alroy H, Gadoth A. Erdheim-Chester disease presenting with chorea and mimicking IgG4-related disorder. Neurol Clin Pract. 2019;9(6):524-526.

22. Liu C, Zhang P, Zhang W. Immunological mechanism of IgG4related disease. J Transl Autoimmun. 2020;3:100047.

23. Arai Y, Yamashita K, Kuriyama K, et al. Plasmacytoid dendritic cell activation and IFN- production are prominent features of murine autoimmune pancreatitis and human IgG4-related autoimmune pancreatitis. J Immunol. 2015;195(7):3033-3044.

24. Cohen-Aubart F, Guerin M, Poupel L, et al. Hypoalphalipoproteinemia and BRAF V600E mutation are major predictors of aortic infiltration in the Erdheim-Chester disease. Arterioscler Thromb Vasc Biol. 2018;38(8):1913-1925.

25. Morimoto A, Oh Y, Nakamura S, et al. Inflammatory serum cytokines and chemokines increase associated with the disease extent in pediatric Langerhans cell histiocytosis. Cytokine. 2017;97:73-79. 